Introduction to Lock-in Detection

Download the Whitepaper

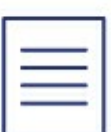

f ( SUBMIT YOUR ARTICLE

AIP Applied Physics Letters

HOME

ISSUES

INFO

FOR AUTHORS

COLLECTIONS

SIGN UP FOR ALERTS

\title{
Lasing in cuprous iodide microwires
}

Appl. Phys. Lett. 111, 031105 (2017); https://doi.org/10.1063/1.4990524

Marcel Wille', (D) Evgeny Krüger ${ }^{1}$, Steffen Blaurock ${ }^{2}$, Vitaly Zviagin ${ }^{1}$, Rafael Deichsel', Gabriele Benndorf ${ }^{1}$, Lukas Trefflich', Volker Gottschalch $^{2}$, Harald Krautscheid ${ }^{2}$, Rüdiger Schmidt-Grund ${ }^{1}$, and (D) Marius Grundmann ${ }^{1}$

View Affiliations

1 PDF

TOPICS
- Excitons
- Crystal structure
- Laser theory
- Semiconductor device
fabrication
- Luminescence
spectroscopy
- Whispering gallery wave
modes
- Cryogenics
- Chemical compounds
- Acoustical properties

\section{ABSTRACT}

We report on the observation of lasing in cuprous iodide (CuI) microwires. A vapor-phase transport growth procedure was used to synthesize $\mathrm{CuI}$ microwires with low defect concentration. The crystal structure of single microwires was determined to be of zincblende-type. The high optical quality of single microwires is indicated by the observed series of excitonic emission lines as well as by the formation of gain under optical excitation. Lasing of triangular whispering-gallery modes in single microwires is demonstrated for fs- and ns-excitation from cryogenic temperatures up to $200 \mathrm{~K}$. Time-resolved microphotoluminescence studies reveal the dynamics of the laser process on the time scale of several picoseconds.

Cuprous iodide (CuI) is a wide bandgap semiconductor which has gained interest in the last few years due to its possible application as a transparent conductive material. Specifically, zincblende-type $\gamma$-CuI, with direct bandgap energies of $3.115 \mathrm{eV}$ at $4 \mathrm{~K}$ and $2.95 \mathrm{eV}$ at $300 \mathrm{~K}, 1,2$ is of 
great interest due to its transparency in the visible spectral range, its intrinsic p-type conductivity, and its high hole-mobility of up to 43 $\mathrm{cm}^{2} / \mathrm{V} \mathrm{s}^{3-5}$ Therefore, CuI is well suited as a p-component in optoelectronic devices, i.e., as a collection layer for holes in solar cells and in transparent $\mathrm{p}-\mathrm{n}$-heterojunctions. ${ }^{6-8}$ Micro- and nanostructures of p-type CuI can be complementary building blocks in compact integrated optoelectronic circuits and devices. In particular, the intense photoluminescence (PL) in the near ultraviolet (NUV) range and the high exciton binding energy of $62 \mathrm{meV}$ support the applicability of $\mathrm{CuI}$ in optoelectronic devices. ${ }^{9}$ However, the synthesis of $\mathrm{CuI}$ micro- and nanostructures and their optical properties are almost unexplored in contrast to other compound semiconductors like zinc oxide ( $\mathrm{ZnO}),{ }^{10-12}$ cadmium sulfide $(\mathrm{CdS}),{ }^{13,14}$ gallium nitride $(\mathrm{GaN}),{ }^{15,16}$ and gallium arsenide $(\mathrm{GaAs}) \cdot{ }^{17,18}$

The aim of this work is to present CuI microstructures as potential candidates for future optoelectronic applications. We applied a vaporphase transport growth process in order to synthesize high quality microstructures of $\mathrm{CuI}$, characterize their morphology and crystal structure properties, and demonstrate their outstanding ability to act as a laser under optical excitation. The temperature limit of the lasing process in single microwires will be investigated. Using the timeresolved micro-photoluminescence $\mu$-PL technique, the dynamics of the lasing emission of single microwires is investigated in order to reveal information about the underlying decay process.

Cuprous iodide microwires with diameters in the range of (0.5-50) $\mu \mathrm{m}$ and lengths up to several millimeters were synthesized using a vaporphase transport growth procedure, similar to the process presented by Goto et al. ${ }^{1}$ The source materials $0.58 \mathrm{~g} \mathrm{CuI} \mathrm{(99.995 \% ,} \mathrm{Carl} \mathrm{Roth)} \mathrm{and} 1.22$ g iodide (99.5\%, Alfa Aesar) were filled into a glass tube (a diameter of 3.5 $\mathrm{cm}$ and a length of $25 \mathrm{~cm}$ ). After cooling the mixed solid powders in liquid nitrogen, the glass tube was evacuated to a pressure of $<10^{-3} \mathrm{mbar}$ and subsequently sealed. The growth of $\mathrm{CuI}$ microwires took place in a threezone-furnace with a linear temperature profile between $250^{\circ} \mathrm{C}$ and 450 ${ }^{\circ} \mathrm{C}$. The glass tube was kept in the furnace for 4 weeks at an inclination angle of $10^{\circ}$. The largest amount of microwires was found in the central part of the glass tube. Scanning electron microscopy (SEM) was used to examine the morphology and structural properties of single microwires. $\mathrm{X}$-ray diffraction (XRD) patterns were recorded in order to study the crystal structure of $\mathrm{CuI}$ microwires. The chemical composition was examined using energy dispersive X-ray spectroscopy (EDX) equipped in 
the SEM system. For subsequent spatially resolved PL investigations, the microwires were transferred onto a clean $\mathrm{SiO}_{2} / \mathrm{Si}$ substrate using an acupuncture needle. The thermally grown low refractive index $\mathrm{SiO}_{2}$-layer (thickness of $1.5 \mu \mathrm{m}, n_{\mathrm{SiO}_{2}}=1.47$ at $3.0 \mathrm{eV}$ photon energy) causes a reduction of leakage of the electromagnetic field into the substrate. ${ }^{14}$ The substrate with the microwire samples was mounted either in a helium bath cryostat with a sample temperature of $2 \mathrm{~K}$ or in a helium flow cryostat in order to perform temperature dependent measurements in the range of (14-300) K. A frequency doubled titaniumsapphire (Ti:Sa) laser $\left(\lambda_{\text {ex }}=364 \mathrm{~nm}, t_{\text {pulse }}=200 \mathrm{fs}\right.$, and $f_{\text {rep }}=76 \mathrm{MHz}$ ) was used as an excitation laser for time-integrated as well as time-resolved PL measurements at $2 \mathrm{~K}$ and at low excitation densities. The PL was spectrally dispersed by a spectrometer (focal length of $320 \mathrm{~mm}, 2400$ lines $/ \mathrm{mm}$ ) and guided either to a CCD camera (spectral resolution of 0.3 $\mathrm{meV}$ ) or to a time-correlated single-photon counting setup with a time resolution of 20 ps (details can be found in Ref. 19). For $\mu$-PL measurements at high excitation densities, a frequency mixed Nd:YAG laser $\left(\lambda_{\mathrm{ex}}=355 \mathrm{~nm}, f_{\text {rep }}=100 \mathrm{~Hz}\right.$, and $\left.t_{\text {pulse }}=10 \mathrm{~ns}\right)$ and the fs-pulsed Ti:Sa laser were used. The laser beam was focused by a $50 \times$ microscope objective to a spot size of (10-50) $\mu \mathrm{m}^{2}$ for the non-resonant excitation of single microwires. The PL was collected by the same objective, dispersed by a spectrometer and guided either to a CCD camera (spectral resolution of $0.5 \mathrm{meV}$ ) or to a streak camera (Hamamatsu C5680) with a time resolution of $2 \mathrm{ps}$. A variable attenuator was used to adjust the excitation density. The laser power was measured using a calibrated $\mathrm{Si}$ diode power meter.

The analysis of the morphology of zincblende-type $\mathrm{CuI}$ indicates different growth kinetics, as already shown by Grundmann et al. ${ }^{20}$ It was found from SEM investigations that larger microwires often exhibit a belt-like shape (see Fig. S1 in the supplementary material). However, smaller microwires crystallize symmetrically to the wire axis and exhibit a rounded triangular cross-section. Figures 1(a) and 1(b) show the SEM images of an exemplary $\mathrm{CuI}$ microwire with triangular shape. This microwire has a length of $49 \mu \mathrm{m}$ and an approximate diameter of $4.0 \mu \mathrm{m}$. The microwire surfaces are smooth and free of any apparent defects. The chemical composition of several single microwires with different crosssection dimensions was analyzed using the EDX technique. The EDX spectra (see Fig. S2 in the supplementary material) reveal the stoichiometric ratio $\mathrm{Cu} / \mathrm{I} \approx 1 / 1$. X-ray diffraction measurements (see Fig. $\mathrm{S} 3$ in the supplementary material) prove that the microwires crystallize 
in the zincblende structure $(\gamma$-CuI). XRD patterns, taken from a side facet of a belt-like microwire, yield the [111]-orientation of zincblende $\mathrm{CuI}$. However, a detailed analysis of the different growth kinetics of the presented microwires is still pending.

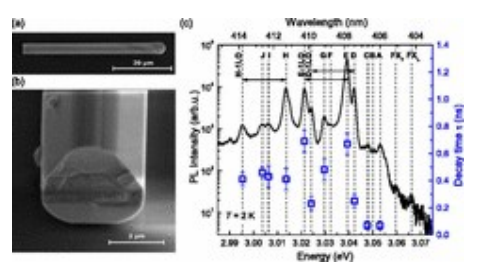

FIG. 1.

(a) SEM image of a Cul microwire with a length of $49 \mu \mathrm{m}$ and a diameter of around $4.0 \mu \mathrm{m}$. The high resolution SEM image in (b) was taken at a tilt angle of $45^{\circ}$ and reveals a rounded triangular microwire cross-section. (c) The PL spectra (Ti:Sa, $P_{\text {ex }}=0.3 \mathrm{~nJ} / \mathrm{cm}^{2}$ ) at a temperature of $2 \mathrm{~K}$ exhibit a series of excitonic emission lines. The energies of the labeled transitions are listed in Table I. Time-resolved PL measurements reveal the respective decay times [blue squares in (d)]. Black arrows represent the energy spacing between an emission line and its LO-phonon replica.

\section{^ PPT | High-resolution}

The PL measurements at low excitation density and $2 \mathrm{~K}$ substrate temperature were performed on six microwires; therefore, both beltlike and triangular microwires have been investigated. All microwires exhibit similar emission characteristics; however, the PL signal from triangular microwires is less intense because of the smaller volume. Figure 1(c) shows the PL spectra from a belt-like microwire with a length of $865 \mu \mathrm{m}$ and cross-section dimensions of $17.9 \mu \mathrm{m} \times 3.8 \mu \mathrm{m}$ (SEM image is shown in Fig. S1 in the supplementary material) for an excitation density of $P_{\mathrm{ex}}=0.3 \mathrm{~nJ} / \mathrm{cm}^{2}\left(1.7 \mathrm{~kW} / \mathrm{cm}^{2}\right)$. The observed excitonic transitions are listed in Table I. The distinct peaks at $3.0662 \mathrm{eV}$ and $3.0596 \mathrm{eV}$ can be assigned to the longitudinal free exciton $\left(\mathrm{FX}_{\mathrm{L}}\right)$ and the transverse free exciton $\left(\mathrm{FX}_{\mathrm{T}}\right)$, respectively. ${ }^{9,21-23}$ The energy spacing of $6.6 \mathrm{meV}$ fits quite well to the known L-T-splitting energy in CuI. ${ }^{23,24}$ A direct comparison of the transitions at lower energies with literature data is rather difficult due to strongly varying emission characteristics depending on the growth conditions, strain, and electronic and structural defects. ${ }^{25,26} \mathrm{~A}$ strong emission in the energy range of the most intense lines D and $\mathrm{E}$ was also observed by Vereshchagin et al. ${ }^{27}$ and was assigned to shallow neutral acceptor transitions. Sauder and Certier $^{28}$ ascribed an intense line in the energy range of the observed emission line $\mathrm{H}$ to the emission 
of excitons bound to neutral acceptors originating from surface near copper vacancies. Note that the emission energy of the bound excitons changes slightly along the microwire axis (maximum deviation of $6 \mathrm{meV}$ for the line $\mathrm{H}$ ). This might be due to the irregular defect incorporation during the growth process. The decay characteristics of the individual excitonic emission lines were investigated with a time-correlated singlephoton counting setup. The characteristic decay times were evaluated by modeling a mono-exponential decay. In the energy range close to the free excitons, the emission decays with a decay time of around $(64 \pm 30)$ ps. With increasing binding energy of the bound excitons, the decay time increases and reaches a value of $(680 \pm 80)$ ps for the emission line E. The assigned phonon replica $\left(E_{\mathrm{LO}} \approx 18.0 \mathrm{meV}\right)$ of the transitions $\mathrm{D}, \mathrm{E}$, and $\mathrm{H}$ showed similar dynamics to the respective zero-phonon-lines. ${ }^{1}$ In contrast, the emission lines I and J, which might be the 2-LO-phonon replica of the lines $\mathrm{D}$ and $\mathrm{E}$, exhibit significantly shorter decay times compared to the respective zero-phonon-lines. This could be artificially caused by the spectral overlap with the intense adjacent transitions $\mathrm{H}$ and $\mathrm{H}-1 \mathrm{LO}$ which have a decay time of approximately $410 \mathrm{ps}$. Hence, the origin of the lines I and J remains unclear.

TABLE I. Emission lines and the respective decay times obtained from a CuI microwire at $2 \mathrm{~K}$.

Excitation density dependent $\mu$-PL measurements were performed on microwires with triangular cross-section. Their smaller dimensions lead to a less pronounced absorptive lasing mode suppression, which is induced by the spatially inhomogeneous excitation and the limited carrier diffusion length. ${ }^{12}$ Figure 2(a) shows the temperature dependent $\mu$-PL spectra from the microwire shown in Fig. 1, at low (dashed lines) and high (solid lines) excitation densities using the ns-pulsed Nd:YAG laser. For low excitation density at $14 \mathrm{~K}$, the emission from bound excitons $(\mathrm{C}-$, D-, E-, and H-bands) and their LO-phonon replica can be observed. Furthermore, an additional band appears in the energy range of (3.0253.031) eV. This band most likely originates from inelastic exciton-exciton scattering (P-band), where the radiatively decaying exciton emits a photon and scatters another exciton into a higher excited state. ${ }^{29}$ Tanaka and Nakayama ${ }^{30}$ demonstrated the P-band-correlated optical gain of $\sim 400 \mathrm{~cm}^{-1}$ in $\mathrm{CuI}$ thin films using the stripe length technique and studied the accompanied stimulated emission. With increasing excitation density, bound exciton related emission saturates. At a certain excitation 
density, narrow emission lines appear in the spectral vicinity of the Pband emission. These narrow lines were observed up to a temperature of $200 \mathrm{~K}$ for an excitation density of $460 \mathrm{~kW} / \mathrm{cm}^{2}$ [solid lines in 2(a)]. Above this critical temperature, a dramatic decrease in PL intensity was observed. With increasing sample temperature, the P-band becomes less pronounced at low excitation density and vanishes at around $150 \mathrm{~K}$. This might be caused by the ineffective scattering of excitons at higher temperatures. As a consequence, P-band correlated gain and thus the lasing process break down above a critical temperature. The excitation density dependent $\mu$-PL spectra at $200 \mathrm{~K}$, using the fs-pulsed Ti:Sa laser as the excitation source, are shown in Fig. 2(b). They clearly demonstrate the transition from broad spontaneous emission to stimulated emission from narrow modes. In order to prove lasing action of single microwires, the input-output characteristic was analyzed. Figure 2(c) depicts the integrated $\mu$-PL intensity of a lasing mode as a function of the excitation density. The double-logarithmic plot shows a distinct S-shaped characteristic which represents the threshold behavior of the lasing transition. The lasing threshold density $P_{\text {th }}=300 \mu \mathrm{J} / \mathrm{cm}^{2}$ was determined by modeling the excitation density dependent integrated $\mu$-PL intensity with a multimodal laser approach. ${ }^{31}$ Below $P_{\mathrm{th}}$, the emission intensity increases linearly with the excitation density. In the threshold regime, the slope becomes strongly nonlinear and tends to saturate in the lasing regime. This observation clearly demonstrates lasing in the investigated microwire. Overcoming a further threshold at around $650 \mu \mathrm{J} / \mathrm{cm}^{2}$, the mode intensity declines reversibly due to the enhancement of modes at lower energies [data shown in dark green in Figs. 2(b) and 2(c)]. Note that we confirmed lasing at temperatures up to $200 \mathrm{~K}$ for several individual microwires with triangular cross-section.

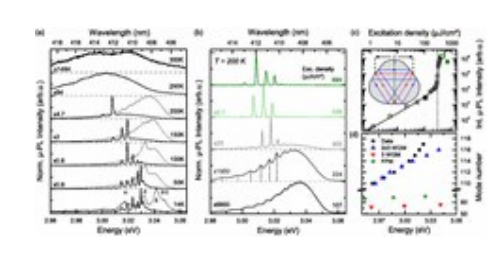

FIG. 2.

(a) The $\mu$-PL spectra (Nd:YAG, $P_{\text {ex }}=$ $460 \mathrm{~kW} / \mathrm{cm}^{2}$ ) from the triangular microwire using ns-excitation reveal a stable lasing process for temperatures up to $200 \mathrm{~K}$ (solid lines). The respective $\mu$-PL spectra below the lasing threshold $\left(P_{\mathrm{ex}} \sim 0.1 P_{\mathrm{th}}\right.$ are depicted as dashed lines. (b) Excitation density dependent $\mu$-PL spectra (Ti:Sa) from the same microwire using fs-excitation reveal a transition from broad spontaneous emission to emission from narrow modes at $200 \mathrm{~K}$. (c) Doublelogarithmic plot of the integrated $\mu-\mathrm{PL}$ intensity of a lasing mode in dependence on the excitation density indicates a lasing threshold 
density $P_{\mathrm{th}} \approx 300 \mu \mathrm{J} / \mathrm{cm}^{2}$. The filled squares correspond to the spectra in (b). The inset in (c) sketches different types of possible resonant modes in the triangular microwire geometry [green: FabryPerót modes (FPM) and red and blue: first- and second-order triangular whispering-gallery modes (3-WGM, $2 \times 3-W G M)$, respectively]. (d) The experimentally deduced mode positions at 200 $\mathrm{K}$ (black squares) compared to theoretical mode positions of FPM (green circles), 3-WGM (red triangles), and $2 \times 3-$ WGM (blue triangles) for a Cul microwire with a dimension $I=3.16 \mu \mathrm{m}$ and a refractive index $n=(2.472 \ldots 2.550)$ obtained from spectroscopic ellipsometry measurements on a Cul thin film (see Fig. S4 in the supplementary material).

\section{^ PPT | High-resolution}

The spectral mode positions were analyzed in order to deduce the dominant mode type of the microwire cavity. Figure 2(d) depicts the experimentally observed mode positions at $200 \mathrm{~K}$. The data were extracted slightly below $P_{\text {th }}$ [dark gray curve in Fig. 2(b)]. The mode spacing increases significantly from around $4.8 \mathrm{meV}$ to around $13.6 \mathrm{meV}$ with decreasing mode energy. For the triangular microwire, we assume three different types of lateral lasing modes: Fabry-Pérot modes (FPM) and triangular whispering-gallery modes (WGM) of first- (3-WGM) and second order $(2 \times 3-$ WGM $)$, as depicted in the inset of Fig. 2(c). A FabryPérot cavity model (green) does not fit the mode positions. A plane wave model for TE-polarized WGM in triangular cavities (analogous to hexagonal cavities by Wiersig $^{32}$ ) predicts

$$
E=\frac{h c}{n L}\left(N+\frac{m}{\pi} \arctan \left(n \sqrt{\frac{n^{2}-4}{3}}\right)\right)
$$

Here, $h$ is Planck's constant, $c$ is the vacuum speed of light, $n$ is the energy dependent refractive index, $L$ is the resonator length, $N$ is the interference order, and $m$ is the number of total internal reflections. For first- and second-order triangular modes, we find $L_{3} \approx 31, m_{3}=3$ and $L_{2 \times 3}$ $\approx 61, m_{2 \times 3}=6$, respectively. From spectroscopic ellipsometry measurements of a CuI thin film (see Fig. S4 in the supplementary material), we obtained the refractive index $n=(2.472 \ldots .2 .550)$ in the spectral range of the observed lasing emission. The predicted mode positions for $2 \times 3$-WGM fit the experimentally determined values quite well, as can be seen in Fig. 2(d). Here, a microwire dimension $l=3.16 \mu \mathrm{m}$ was used, which corresponds well to the SEM data. These findings 
indicate strongly that the triangular microwire acts as a whisperinggallery type resonator for second-order triangular modes. The slight deviations in the vicinity of the exciton resonance might arise from the differences between the refractive indices of the measured thin film and the microwires.

The dynamics of the microwire lasers was investigated in the temperature range of (14-200) K using a streak camera. Figure 3(a) shows the time-resolved spectra of the lasing emission from the microwire for an excitation density of $2.5 P_{\text {th }}$ at $14 \mathrm{~K}$. The transient of the most intense lasing mode is shown in Fig. 3(b). Modeling the decay of this lasing mode with a mono-exponential function yields the characteristic decay time $\tau_{\mathrm{L}}$ $=(6.7 \pm 2.0) \mathrm{ps}$ in the lasing regime. Upon variation of the excitation density from $P_{\text {th }}$ to $2.5 P_{\text {th }}, \tau_{\mathrm{L}}$ fluctuates statistically in the range of (3.58.5) ps independent of the sample temperature. The small decay time $\tau_{\mathrm{L}}$ reflects the ultrafast recombination after the strong excitation pulse. Ichida et al. ${ }^{33}$ studied the optical gain in CuI thin films and determined a similar decay time of 8 ps under high optical excitation. They ascribed this fast recombination process to an effective scattering of excitons (Pband). The emission energy and the slight red-shift for higher excitation densities agree with the results found here. However, the formation of an electron-hole-plasma would also cause sufficiently high gain to overcome the resonator losses, evoke a red-shift with increasing excitation density, and cause similar characteristic decay times in the lasing regime, like in other semiconductors. ${ }^{34}$ Hence, further investigations are necessary to understand the gain mechanism of the observed lasing in CuI microwires.

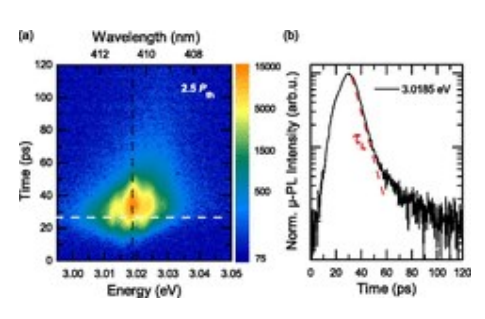

FIG. 3.

(a) Time-resolved $\mu$-PL spectra from the triangular microwire at $14 \mathrm{~K}$ (Ti:Sa, $2.5 P_{\text {th) }}$. Note the logarithmic color scale. The dashed white line indicates the impingement of the excitation laser pulse. (b) Normalized transient of the most intense lasing mode extracted at the energy $3.0185 \mathrm{eV}$ (indicated by the black dashed line in (a)) exhibits the characteristic decay time $\tau_{\mathrm{L}}=(6.7 \pm 2.0)$ ps.

\section{^ PPT | High-resolution}

In summary, we have synthesized [111]-oriented zincblende-type CuI 
microwires by applying a vapor-phase transport growth procedure. Their outstanding optical properties constitute in a series of excitonic emission lines at low excitation density conditions. Time-resolved PL measurements at $2 \mathrm{~K}$ revealed the decay times of individual free and bound excitons. We demonstrated the observation of lasing of secondorder triangular modes in $\mathrm{CuI}$ microwires with triangular cross-section for ns- and fs-pulsed laser excitation with threshold densities of 400 $\mathrm{kW} / \mathrm{cm}^{2}$ and $300 \mu \mathrm{J} / \mathrm{cm}^{2}\left(1.5 \mathrm{GW} / \mathrm{cm}^{2}\right)$, respectively. We showed that the lasing process is stable up to a temperature of $200 \mathrm{~K}$. The lasing emission was observed in the energy range where the exciton-exciton scattering related P-band is expected. The dynamics of the microwire lasing emission was investigated for different temperatures as well as excitation densities and revealed a characteristic decay time $\tau_{\mathrm{L}}=(3.5-8.5)$ ps.

See supplementary material, which contains the experimental results of the EDX, XRD, and ellipsometry measurements as well as a description of the respective techniques.

This work was supported by the Deutsche Forschungsgemeinschaft within Gr 1011/26-1 and through FOR1616, by the "Leipzig Graduate School of Natural Sciences - BuildMoNa" and by Universität Leipzig in the framework of research profile area "Complex Matter." We thank M. Kneiß, C. Sturm, and B. Rheinländer for valuable discussions and C. Yang for the growth of $\mathrm{CuI}$ thin films for ellipsometry measurements.

\section{REFERENCES}

1. T. Goto, T. Takahashi, and M. Ueta, J. Phys.

Soc. Jpn. 24, 314 (1968).

https://doi.org/10.1143/JPSJ.24.314, Google Scholar, Crossref, CAS

2. S. Ves, D. Glötzel, M. Cardona, and H.

Overhof, Phys. Rev. B 24, 3073 (1981).

https://doi.org/10.1103/PhysRevB.24.3073, Google Scholar, Crossref, CAS

3. J. Wang, J. Li, and S.-S. Li, J. Appl. Phys. 110, 054907 (2011). 
4. C. Yang, M. Kneiß, M. Lorenz, and M. Grundmann, Proc. Natl. Acad. Sci. U.S.A. 113, 12929 (2016).

https://doi.org/10.1073/pnas.1613643113, Google Scholar, Crossref, CAS

5. D. Chen, Y. Wang, Z. Lin, J. Huang, X. Chen, D. Pan, and F. Huang, Cryst. Growth Des. 10, 2057 (2010). https://doi.org/10.1021/cg100270d, Google Scholar, Crossref, CAS

6. J. A. Christians, R. C. Fung, and P. V. Kamat, J. Am. Chem. Soc. 136, 758 (2013). https://doi.org/10.1021/ja411014k, Google Scholar, Crossref

7. F.-L. Schein, H. von Wenckstern, and M. Grundmann, Appl. Phys. Lett. 102, 092109 (2013). https://doi.org/10.1063/1.4794532, Google Scholar, Scitation

8. C. Yang, M. Kneiß, F.-L. Schein, M. Lorenz, and M. Grundmann, Sci. Rep. 6, 21937 (2016) https://doi.org/10.1038/srep21937. Google Scholar, Crossref, CAS

9. S. Nikitine, Philos. Mag. 4, 1 (1959). https://doi.org/10.1080/14786435908238225 , Google Scholar, Crossref, CAS

10. M. Huang, S. Mao, H. Feick, H. Yan, Y. Wu, H. Kind, E. Weber, R. Russo, and P. Yang, Science 292, 1897 (2001).

https://doi.org/10.1126/science.1060367, Google Scholar, Crossref, CAS

11. C. Czekalla, T. Nobis, A. Rahm, B. Cao, J. 
Zúñiga-Pérez, C. Sturm, R. Schmidt-Grund, M. Lorenz, and M. Grundmann, Phys. Status Solidi B 247, 1282 (2010).

https://doi.org/10.1002/pssb.200945527, Google Scholar, Crossref, CAS

12. M. Wille, T. Michalsky, E. Krüger, M.

Grundmann, and R. Schmidt-Grund, Appl.

Phys. Lett. 109, 061102 (2016).

https://doi.org/10.1063/1.4960660, Google Scholar, Scitation

13. R. Agarwal, C. Barrelet, and C. Lieber, Nano Lett. 5, 917 (2005).

https://doi.org/10.1021/nl050440u, Google Scholar, Crossref, CAS

14. R. Röder, M. Wille, S. Geburt, J. Rensberg, M. Zhang, J. Lu, F. Capasso, R. Buschlinger, U. Peschel, and C. Ronning, Nano Lett. 13, 3602 (2013). https://doi.org/10.1021/nl401355b, Google Scholar, Crossref

15. J. Johnson, H.-J. Choi, K. Knutsen, R. Schaller, P. Yang, and R. Saykally, Nat. Mater. 1, 106 (2002). https://doi.org/10.1038/nmat728, Google Scholar, Crossref, CAS

16. C. Tessarek, R. Röder, T. Michalsky, S. Geburt, H. Franke, R. Schmidt-Grund, M. Heilmann, B. Hoffmann, C. Ronning, M. Grundmann et al., ACS Photonics 1, 990 (2014). https://doi.org/10.1021/ph500220v, Google Scholar, Crossref, CAS

17. X. Duan, J. Wang, and C. M. Lieber, Appl. Phys. Lett. 76, 1116 (2000). https://doi.org/10.1063/1.125956, Google Scholar, Scitation, CAS 
18. D. Saxena, S. Mokkapati, P. Parkinson, N. Jiang, Q. Gao, H. Tan, and C. Jagadish, Nat. Photonics 7, 963 (2013).

https://doi.org/10.1038/nphoton.2013.303, Google Scholar, Crossref, CAS

19. A. Müller, M. Stölzel, C. Dietrich, G.

Benndorf, M. Lorenz, and M. Grundmann, J.

Appl. Phys. 107, 013704 (2010).

https://doi.org/10.1063/1.3270431,

Google Scholar, Scitation

20. M. Grundmann, F.-L. Schein, M. Lorenz, T.

Böntgen, J. Lenzner, and H. von Wenckstern,

Phys. Status Solidi A 210, 1671 (2013).

https://doi.org/10.1002/pssa.201370056,

Google Scholar, Crossref, CAS

21. V. Nikitenko, S. Stoyukhin, V. Popolitov, and

Y. M. Mininzon, J. Appl. Spectrosc. 34, 410

(1981). https://doi.org/10.1007/BF00614221,

Google Scholar, Crossref

22. T. Sauder, A. Daunois, J. Deiss, and J. Merle,

Solid State Commun. 51, 323 (1984).

https: / /doi.org/10.1016/0038-1098(84)90697

$-5$

, Google Scholar, Crossref, CAS

23. J. Serrano, C. Schweitzer, C. Lin, K. Reimann,

M. Cardona, and D. Fröhlich, Phys. Rev. B 65,

125110 (2002).

https://doi.org/10.1103/PhysRevB.65.125110,

Google Scholar, Crossref

24. S. Suga, K. Cho, Y. Niji, J. Merle, and T.

Sauder, Phys. Rev. B 22, 4931 (1980).

https://doi.org/10.1103/PhysRevB.22.4931,

Google Scholar, Crossref, CAS 
25. P. Gao, M. Gu, X. Liu, Y.-Q. Zheng, and E. Shi, Optik 125, 1007 (2014).

https://doi.org/10.1016/j.ijleo.2013.07.112, Google Scholar, Crossref, CAS

26. G. Lin, F. Zhao, Y. Zhao, D. Zhang, L. Yang, X. Xue, X. Wang, C. Qu, Q. Li, and L. Zhang, Materials 9, 990 (2016). https://doi.org/10.3390/ma9120990, Google Scholar, Crossref

27. I. Vereshchagin, V. Nikitenko, and S.

Stoyukhin, J. Lumin. 29, 215 (1984).

https://doi.org/10.1016/S0022-2313(84)9008

8-7

, Google Scholar, Crossref, CAS

28. T. Sauder and M. Certier, Phys. Lett. A 101, 55 (1984).

https://doi.org/10.1016/0375-9601(84)90092

$-6$

, Google Scholar, Crossref

29. C. Klingshirn and H. Haug, Phys. Rep. 70, 315 (1981).

https://doi.org/10.1016/0370-1573(81)90190-

3

, Google Scholar, Crossref, CAS

30. I. Tanaka and M. Nakayama, J. Appl. Phys. 92, 3511 (2002).

https://doi.org/10.1063/1.1502205, Google Scholar, Scitation, CAS

31. L. Casperson, J. Appl. Phys. 46, 5194 (1975).

https://doi.org/10.1063/1.321311,

Google Scholar, Scitation, CAS

32. J. Wiersig, Phys. Rev. A 67, 023807 (2003).

https://doi.org/10.1103/PhysRevA.67.023807, 
33. H. Ichida, Y. Kanematsu, T. Shimomura, K.

Mizoguchi, D. Kim, and M. Nakayama, Phys.

Rev. B 72, 045210 (2005).

https://doi.org/10.1103/PhysRevB.72.045210,

Google Scholar, Crossref

34. K. Bohnert, G. Schmieder, and C. Klingshirn, Phys. Status Solidi B 98, 175 (1980).

https://doi.org/10.1002/pssb.2220980117,

Google Scholar, Crossref, CAS

Published by AIP Publishing.

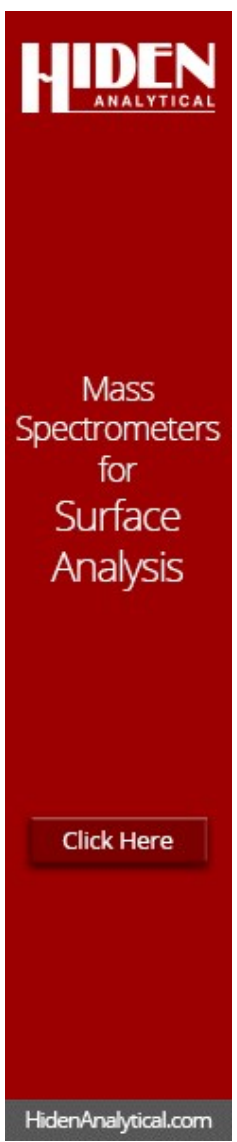




\begin{tabular}{|l|l|l|}
\hline AUTHOR & ABOUT & PRIVACY POLICY \\
LIBRARIAN & CONTACT & TERMS OF USE \\
\hline ADVERTISER & HELP & FOLLOW AIP PUBLISHING: \\
\hline This website uses cookies to ensure the best user experience. Learn more \\
\hline
\end{tabular}

